ACTA MYCOLOGICA

Vol. 42 (2): 287-290

2007
Dedicated to Professor Alina Skirgietto

on the occasion of her ninety-fifth birthday

\title{
Rinodina griseosoralifera, a lichen species new to the Western Carpathians
}

\author{
PAWEL CZARNOTA ${ }^{1}$ and MARTIN KUKWA ${ }^{2}$
}

${ }^{1}$ Scientific Laboratory of the Gorce National Park, Poręba Wielka 590

PL-34-735 Niedźwiedź, pawel.czarnota@gpn.pl

${ }^{2}$ Department of Plant Taxonomy and Nature Protection, University of Gdańsk

Al. Legionów 9, PL-80-441 Gdańsk, dokmak@univ.gda.pl

Czarnota P., Kukwa M.: Rinodina griseosoralifera, a lichen species new to the Western Carpathians. Acta Mycol. 42 (2): 287-290, 2007.

Rinodina griseosoralifera Coppins is reported for the first time from Poland and the Western Carpathians. It is known there from the Gorce Mts, only locality up to now. Details of the chemistry, morphology and general distribution are provided and similar taxa are discussed.

Key words: Rinodina, sorediate lichens, atranorin, zeorin, Carpathians, Poland

\section{INTRODUCTION}

Lichen genus Rinodina (Ach.) Gray is comprised of crustose lichens with lecanorine apothecia and brown, characteristically septate spores (for full description see Nowak 1998; Mayrhofer, Moberg 2002). Most of the taxa reproduce by spores, but a few possess vegetative soredia and isidia; these are usually sterile and usually difficult to identify since many other, even unrelated, species are superficially similar. In such cases, secondary chemistry is invaluable in their determination (e.g. Tønsberg 1992).

In Poland, only two sorediate epiphytic Rinodina species, $R$. colobina (Ach.) Th. Fr. and R. efflorescens Malme, have been reported to date (Fałtynowicz 2003). During the identification of some sterile crustose lichens we came across a specimen resembling $R$. efflorescens, but thin layer chromatography revealed the presence of atranorin and zeorin. The chemistry and morphology matches $R$. griseosoralifera Coppins, a taxon not known from our country.

This paper presents the first record of $R$. griseosoralifera for Poland and the Western Carpathians, provides information on its chemistry, morphology and general distribution, and discusses its affinities to several sorediate, usually sterile, taxa. 


\section{MATERIAL AND METHODS}

Morphology of the specimen was studied with the aid of a stereomicroscope and chemical analyses were carried out using thin layer chromatography (TLC) according to Orange et al. (2001). The material is stored in GPN, with a duplicate donated to UGDA-L.

\section{RESULTS}

\section{Rinodina griseosoralifera Coppins - Lichenologist 21: 169. 1989}

Morphology AND CHEMISTRY. $R$. griseosoralifera is a crustose lichen forming an episubstratal thallus and consisting of greenish-white to brown areoles which usually entirely dissolve into soredia, as in the Polish collection. Soralia are blue-grey, formed at the top of areoles, discrete (Fig. 1A) or forming a more or less continuous sorediate crust (Fig. 1B). Apothecia were not observed in the Polish collection.

The species produces atranorin and zeorin. Tønsberg (1992) also reported possible traces of unidentified terpenoids, but we did not detect any additional substances.

Affinities. Due to its blue-grey external soredia $R$. griseosoralifera is superficially similar to several taxa: Buellia griseovirens (Turner \& Borrer ex Sm.) Almb., Caloplaca chlorina (Flot.) Sandst., Rinodina colobina and R. efflorescens. The most significant character is the chemistry. B. griseovirens produces norstictic acid and atranorin as major compounds (squash preparation reacts $\mathrm{K}+$ yellow turning orange and forming needle-like crystals), $C$. chlorina does not contain lichen substances, $R$. colobina has substances called 'colobina unknows' in the thallus, and $R$. efflorescens has pannarin (with additional substances) causing $\mathrm{P}+$ orange-red reaction of soralia (Tønsberg 1992). Additional characters differentiating these taxa are shown in the Table 1.

Ecology. In the Polish locality the species covered eutrophic bark and occasionally mosses at the base of an old Pyrus, forming large patches up to ca $10 \mathrm{dm}^{2}$. It was associated with Acrocordia gemmata (Ach.) A. Massal., Bacidia subincompta (Hoffm.) A. Massal., Caloplaca obcurella (J. Lahm) Th. Fr., Candelariella xanthostigma (Ach.) Lettau, Lepraria vouauxii (Hue) R. C. Harris, Normandina pulchella (Borrer) Nyl. and Phaeophyscia endophoenicea (Harm.) Moberg. The Polish habitat corresponds to that described by Palice (1999), suggesting that it may by a more frequent species in old orchards or solitary trees in Poland, but overlooked due to its usually sterile form and the necessity to examine it by TLC.

Distribution. In Poland, $R$. griseosoralifera is known from a single locality in the Gorce Mts, but it may be more widely distributed since suitable habitats are common in the country.

In Europe, the species is rather uncommon, but widely distributed. To date, it has been reported from Austria and Britain (Coppins 1989), Ireland (Seaward 1994), Czech Republic (Palice 1999), Germany (Scholz 2000), the Netherlands (Aptroot et al. 2001), Norway (Coppins 1989; Tønsberg 1992), Spain (Llimona, Hladun 2001), Switzerland (Dietrich, Scheidegger 1996) and Ukraine (Eastern 
Table 1

Comparison of Rinodina griseosoralifera and superficially similar taxa occurring in Poland (acc. to Tønsb e r 1992 and author's studies)

\begin{tabular}{|c|c|c|c|c|c|}
\hline \multirow[b]{2}{*}{ Feature } & \multicolumn{5}{|c|}{ Taxa } \\
\hline & $\begin{array}{l}\text { Rinodina grise- } \\
\text { osoralifera }\end{array}$ & $\begin{array}{l}\text { Rinodina ef- } \\
\text { florescens }\end{array}$ & $\begin{array}{l}\text { Rinodina } \\
\text { colobina }\end{array}$ & $\begin{array}{l}\text { Caloplaca } \\
\text { chlorina }\end{array}$ & $\begin{array}{l}\text { Buellia griseo- } \\
\text { virens }\end{array}$ \\
\hline $\begin{array}{l}\text { Non-soraliate } \\
\text { parts of thallus }\end{array}$ & $\begin{array}{c}\text { indistinct, } \\
\text { small areolae, } \\
\text { early dissolv- } \\
\text { ing into soralia } \\
\text { or sorediate } \\
\text { crust }\end{array}$ & $\begin{array}{l}\text { areolate to } \\
\text { subsqua- } \\
\text { mulose, often } \\
\text { dissolving into } \\
\text { soralia }\end{array}$ & $\begin{array}{l}\text { areolate to } \\
\text { warted, } \pm \text { dis- } \\
\text { appearing, } \\
\text { early becom- } \\
\text { ing sorediate }\end{array}$ & $\begin{array}{l}\text { mostly quite } \\
\text { thick, at least } \\
\text { partially dis- } \\
\text { tinctly areolate } \\
\text { to } \pm \text { leprose }\end{array}$ & $\begin{array}{c}\text { mostly distinct, } \\
\text { areolate to } \\
\text { more or less } \\
\text { continuous }\end{array}$ \\
\hline Prothallus & $\begin{array}{l}\text { indistinct, } \\
\text { brown visible } \\
\text { around the } \\
\text { areolae }\end{array}$ & $\begin{array}{l}\text { indistinct, be- } \\
\text { tween areolae, } \\
\text { sometimes } \\
\text { brownish black }\end{array}$ & absent & $\begin{array}{l}\text { sometimes dis- } \\
\text { tinct, brownish } \\
\text { to brownish } \\
\text { violet }\end{array}$ & $\begin{array}{l}\text { usu- } \\
\text { ally present, } \\
\text { brownish, } \\
\text { often bluish } \\
\text { tinged }\end{array}$ \\
\hline $\begin{array}{l}\text { Colour of } \\
\text { soralia }\end{array}$ & $\begin{array}{l}\text { bluish-grey to } \\
\text { pale greyish } \\
\text { green }\end{array}$ & $\begin{array}{l}\text { brown, greyish } \\
\text { brown, pale to } \\
\text { dull greenish } \\
\text { yellow }\end{array}$ & $\begin{array}{l}\text { grey-black to } \\
\text { bluish-grey, } \\
\text { rarely greyish } \\
\text { green }\end{array}$ & $\begin{array}{c}\text { grey to bluish } \\
\text { grey }\end{array}$ & $\begin{array}{l}\text { greyish white, } \\
\text { yellowish, } \\
\text { greenish to } \\
\text { dark grey, } \\
\text { often with a } \\
\text { bluish tinge }\end{array}$ \\
\hline $\begin{array}{l}\text { Shape of } \\
\text { soralia }\end{array}$ & $\begin{array}{l} \pm \text { circular, } \\
\text { plane to con- } \\
\text { vex, discrete } \\
\text { or confluent, } \\
\text { bursting from } \\
\text { areolae }\end{array}$ & $\begin{array}{c}\text { punctiform, } \\
\pm \text { flat to } \\
\text { hemispherical } \\
\text { bursting from } \\
\text { areolae or } \\
\text { substratum }\end{array}$ & $\begin{array}{l}\text { not well de- } \\
\text { fined, mostly } \\
\text { irregular and } \\
\text { efflorescent, } \\
\text { sometimes } \\
\text { marginal at } \\
\text { areolae }\end{array}$ & $\begin{array}{c}\text { marginal } \\
\text { at areolae, } \\
\text { punctiform } \\
\text { to confluent } \\
\text { forming lep- } \\
\text { rose sorediate } \\
\text { crust }\end{array}$ & $\begin{array}{l}\text { mostly circu- } \\
\text { lar, convex, } \\
\text { plane or cra- } \\
\text { teriform }\end{array}$ \\
\hline $\begin{array}{l}\text { Chemistry } \\
\text { (major com- } \\
\text { pounds) }\end{array}$ & $\begin{array}{c}\text { atranorin, } \\
\text { zeorin; K-, } \\
\text { P- or } \pm \text { yellow- } \\
\text { ish, C- }\end{array}$ & $\begin{array}{l}\text { pannarin, } \\
\text { efflorescens } \\
\text { unknown } \\
\text { (pigment), } \\
\text { 土zeorin; } \\
\text { K-, P+ red, } \\
\text { C- }\end{array}$ & $\begin{array}{c}\text { 'colobina } \\
\text { unknowns'; } \\
\text { at least partial- } \\
\text { ly } \mathrm{K} \pm \text { violet, } \\
\mathrm{P}-, \mathrm{C}-\end{array}$ & $\begin{array}{c}\text { no substances; } \\
\text { at least partial- } \\
\text { ly } \mathrm{K} \pm \text { violet, } \\
\mathrm{N} \pm \text { violet, } \mathrm{P}- \\
\mathrm{C}-\end{array}$ & 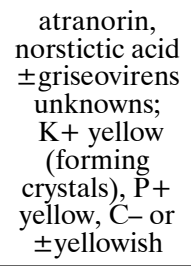 \\
\hline $\begin{array}{l}\text { Pigmentation } \\
\text { of external } \\
\text { soredia }\end{array}$ & $\begin{array}{l}\text { bluish-grey, } \\
\mathrm{K}+\text { brown, N- }\end{array}$ & $\begin{array}{l}\text { often brown, } \\
\mathrm{K}+\text { fuscous } \\
\text { brown, } \mathrm{N}-\end{array}$ & 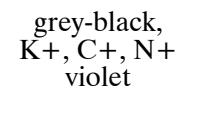 & $\begin{array}{c}\text { bluish, } \mathrm{K}+ \\
\text { violet, } \mathrm{N}+ \\
\text { violet }\end{array}$ & $\begin{array}{l}\text { brown, often } \\
\text { bluish tinged, } \\
\mathrm{K}+\text { fuscous } \\
\text { brown, N- }\end{array}$ \\
\hline
\end{tabular}

Carpathians) (Kondratyuk, Coppins 2000). Outside Europe, it is known from the Canary Islands (Tønsberg 2002) and North America (Tønsberg 1993).

Polish SPecimen eXamined. Western Beskidy Mts, Gorce Mts, Poręba Wielka vil-

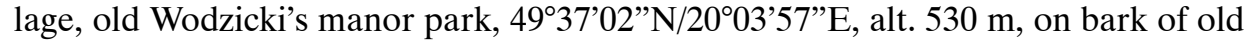
Pyrus communis in old fruit orchard, 18.04.2007, leg. P. Czarnota 5147 (GPN, duplicate in UGDA-L-14058).

Additional specimen examined. Norway, Hordaland, Bergen, Arna, Arna Church, UTM 32V LN 0503, alt. $10 \mathrm{~m}$, on Acer platanoides, 22.07.1990, leg. T. Tønsberg 13338 (BG L-24513). 
Acknowledgements. We are indebted to an anonymous reviewer for suggestions on the manuscript, as well as to Professor Mark Seaward (Bradford, UK) for English correction and some improvements.

\section{REFERENCES}

Aptroot A., Sparrius L., van Herk K., de Bruyn U. 2001. Origin and distribution of recently described lichens from the Netherlands. Aktuelle Lichenologische Mitteilungen 5: 13-25.

Coppins B.J. 1989. Rinodina griseosoralifera, a new corticolous sorediate lichen from western Europe. Lichenologist 21: 169-172.

Dietrich M., Scheidegger C. 1996. The importance of sorediate crustose lichens in the epiphytic lichen flora of the Swiss Plateau and the Pre-Alps. Lichenologist 28: 245-256.

Fałtynowicz W. 2003. The lichens, lichenicolous and allied fungi of Poland - an annotated checklist. W. Szafer Institute of Botany, Polish Academy of Sciences, Kraków, 435 pp.

Kondratyuk S.Ya., Coppins B.J. 1999. Basement for the lichen monitoring in Uzhansky National Nature Park, Ukrainian part of the Biosphere Reserve 'Eastern Carpathians'. Roczniki Bieszczadzkie 8: 149-192.

Llimona X., Hladun N.L. 2001. Checklist of the lichens and lichenicolous fungi of the Iberian Peninsula and Balearic Islands. Bocconea 14: 1-581.

Mayrhofer H., Moberg R. 2002. Rinodina. Nordic Lichen Flora 2: 41-69.

Nowak J. 1998. Porosty (Lichenes). VI. 2. Buelliaceae. (In:) Rośliny zarodnikowe Polski i ziem ościennych. Instytut Botaniki im. W. Szafera PAN, Kraków, 236 pp.

Orange A., James P.W., White F.J. 2001. Microchemical methods for the identification of lichens. British Lichen Society, London, 101 pp.

Palice Z. 1999. New and noteworthy records of lichens in the Czech Republic. Preslia 71: 289-336.

Scholz P. 2000. Katalog der Flechten und flechtenbewohnenden Pilze Deutschlands. Schriftenreihe für Vegetationskunde 31: 1-298.

Seaward M.R.D. 1994. Vice-county distribution of Irish lichens. Proceedings of the Royal Irish Academy 94 B: 177-194.

Tønsberg T. 1992. The sorediate and isidiate, corticolous, crustose lichens in Norway. Sommerfeltia 14: $1-331$.

Tønsberg T. 1993. Additions to the lichen flora of North America. Bryologist 96: 138-141.

Tønsberg T. 2002. Halecania viridescens and Rinodina griseosoralifera new to Africa from the Canary Islands. Graphis Scripta 13 (2): 52-54.

\section{Rinodina griseosoralifera, gatunek porostu nowy dla Karpat Zachodnich}

\section{Streszczenie}

Rinodina griseosoralifera jest nadrzewnym porostem skorupiastym wytwarzającym soredia. Plecha tego gatunku składa się z areolek, z których większość przekształca się w oddzielone od siebie (Fig. 1A) lub zlewające się (Fig. 1B) soralia; soredia mają kolor niebiesko-szary. Obecność wtórnych metabolitów, atranoryny i zeoryny, pozwala odróżnić ten gatunek od innych, morfologicznie bardzo podobnych 'sorediowanych' przedstawicieli rodzaju Rinodina, tj. $R$. colobina i $R$. efflorescens, jak również od Buellia griseovirens i nadrzewnych form Caloplaca chlorina. Cechy chemiczne i morfologiczne ułatwiające identyfikację sterylnych form tych pięciu gatunków przedstawiono w tabeli 1.

$R$. griseosoralifera jest nowym składnikiem bioty porostów Polski i jednocześnie Karpat Zachodnich. Pierwsze jego stanowisko zostało znalezione w Gorcach, w starym sadzie podworskim we wsi Poręba Wielka. Gatunek ten rośnie na korze Pyrus communis, a towarzyszą mu m.in. Acrocordia gemmata, Bacidia subincompta, Caloplaca obscurella, Normandina pulchella i Phaeophyscia endophoenicea. 

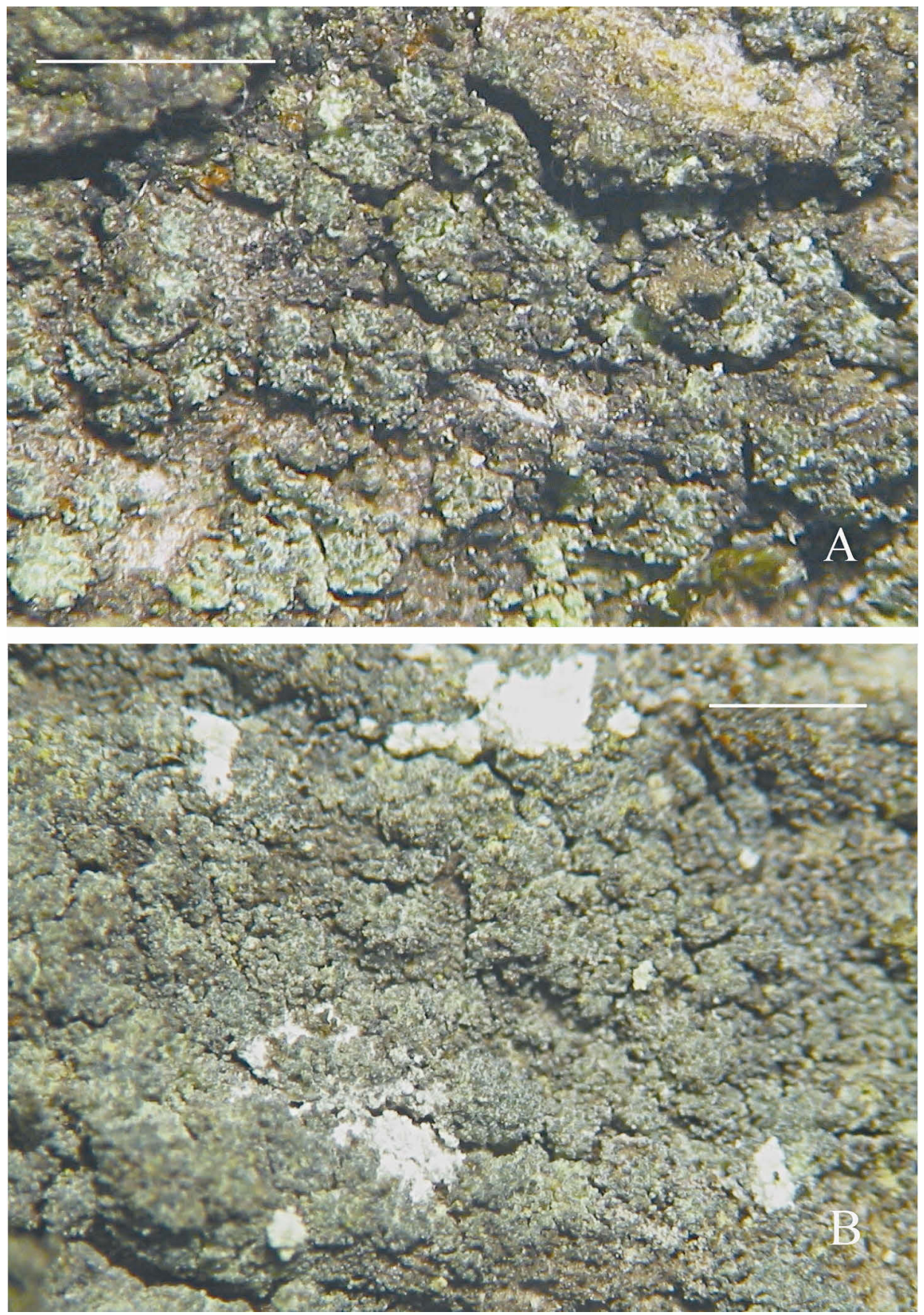

Fig. 1. Habit of Rinodina griseosoralifera (Czarnota 5147, GPN); A - distinct soralia; $\mathrm{B}-$ confluent soralia; scale bars $=1 \mathrm{~mm}$. 